\title{
Antimicrobial resistance profile of Staphylococcus aureus isolates isolated from ear discharges of patients at University of Hawassa comprehensive specialized hospital
}

\author{
Serawit Deyno ${ }^{* *}$, Alemayehu Toma ${ }^{1}$, Mesfin Worku² and Muluken Bekele ${ }^{3}$
}

\begin{abstract}
Background: Drug-resistant microorganism are a growing global danger. Strains of S. aureus have developed resistance to many commonly used antimicrobials due to indiscriminate use of antimicrobials, and treatment becoming a challenge. Studies assessing pattern and determinants of S. aureus resistance in ear infection in Ethiopia are very scarce. This study presents overview of pattern and determinants $\mathbf{S}$. aureus resistance from samples of ear discharge in Ethiopia.

Method: A prospective cross-sectional study was conducted on patients who visit ENT clinic of University of Hawassa comprehensive specialized hospital from February 1, 2016 to November 1, 2016. 117 specimens were collected with sterile applicator cotton swab from 117 patients with ear discharge visiting the clinic. Data was fed and then edited, cleared, entered and analyzed using SPSS version 20.

Result: The prevalence of S.aureus infection was 28.2\%. S. aureus isolates revealed up to 97.0\% level of resistance pattern to the antimicrobials tested. In the determination of the susceptibility of $S$. aureus on nine selected antibiotics by disk diffusion technique, $97.0 \%$ of the isolates were resistant to cloxacilin and $74.2 \%$ showed resistance to vancomycin. The overall rate of MDR was $100 \%$, all of the isolates were found to be resistant to more than two tested antimicrobials.

Conclusion: S. aureus has gotten frighteningly resistant to many of common antimicrobials. Resistance rate to vancomyin is terrifyingly high. It urges us to take measures to delay resistance. Emergence of resistance highlights the value of prudent prescribing of antimicrobials and avoiding their irrational use. Further researches focusing on identifying dynamics promoting resistance, identifying high risk strains and molecular basis of resistance are required.
\end{abstract}

Keywords: Antimicrobial resistance, Staphylococcus aureus, Ethiopia

\footnotetext{
* Correspondence: dserawit@gmail.com

'Department of Pharmacology, School of Medicine, College of Medicine and

Health Sciences, University of Hawassa, P. O. Box 1560, Hawassa, Ethiopia

Full list of author information is available at the end of the article
} 


\section{Background}

Drug-resistant microorganisms are a growing danger to the global society. They endanger people in prosperous societies to poor nations. Antimicrobial resistance (AMR) is a long-standing problem with magnitude and speed it spreads becoming global most serious current public health problem [1-3].

The human Kind was overwhelmed by infectious diseases until the discovery of antimicrobials in the middle of twentieth century. Owing to discovery of various antimicrobial agents the ability to manage infectious diseases has greatly improved [4]. However, the beginning of the era of AMR were recorded soon after the discovery of penicillin, in which a number of treatment failures and occurrence of some bacteria no longer sensitive to penicillin started being noticed [5].

Microbial infection involving microorganisms poses a very serious public health problem all over the world especially in resource limited African countries. Among the many bacterial infections, $S$. aureus is the leading cause of nosocomial infections by gram-positive bacteria[6]. It is notoriously resistant to penicillin and many other antimicrobials [7].

Staphylococci resides with skin, glands and mucous membranes of almost all the warm blooded animals [8]. They prefer aerobic environment but can also grow in the absence of oxygen; range of temperature for growth is $6-44{ }^{\circ} \mathrm{C}$ (optimum $37{ }^{\circ} \mathrm{C}$ ) and the range of $\mathrm{pH}$ is $4.2-$ 9.3 (optimum 7). To date, there are 32 species and eight sub-species in the genus Staphylococcus, many of which preferentially colonize the human body, however, $S$. aureus and S. epidermidis are the two most characterized and studied strains $[9,10]$.

Strains of S.aureus have developed resistance to many commonly used antimicrobial due to indiscriminate use. Staphylococcal resistance to penicillin is mediated by $\beta$-lactamase production. First report of a penicillinresistant strain of $S$. aureus was published in 1945, revealing its association with $\beta$-lactamase enzyme produced by the bacteria. The methicillin resistant staphylococcus aureus (MRSA) is a specific strain of the S. aureus bacterium that has developed antimicrobial resistance to all penicillin's, including methicillin and other narrow-spectrum $\beta$-lactamase-resistant penicillin antimicrobials [11].

Soon after methicillin was introduced into human medicine MRSA was observed [12]. MRSA has since emerged as a serious concern in human medicine. Although these organisms cause the same types of infections as other S. aureus, hospital associated strains have become resistant to most common antimicrobials, making treatment a great challenge.

Ear infection is a common clinical problem throughout the world and the major cause of preventable hearing loss in the developing world [13]. Microbial agents can infect the middle and external parts of the ear and may involve the skin, cartilage, periosteum, ear canal, and tympanic and mastoid cavities [14].About 65-330 million people suffer from ear infection worldwide and $60 \%$ of them had significant hearing loss [15]. The health and economic burden of ear infection is severe especially in Africa and other developing countries where the disease prevalence is high [16]. According to WHO report, Ethiopia belongs among the high ear infection burden countries [16].

It is a common tradition in Ethiopia that antibiotics can be obtained without prescription. This misuse of antibiotics by the public contributes to the emergence and spread of AMR rendering ear infection untreatable [17]. However, studies assessing pattern and determinant of S. aureus resistance in ear infection in Ethiopia are very scarce. This study presents the overview of pattern and determinants S. aureus resistance from samples of ear discharge in Ethiopia.

\section{Methods}

Study setting and study period: This study was conducted at University of Hawassa Comprehensive Specialized Hospital from February 1, 2016 to November 1, 2016.

Study population and Study design: The source population was patients visiting ear, nose, and throat (ENT) clinic at University of Hawassa Comprehensive Specialized Hospital. The study population was patients who had suspected ear infection in Ear, Nose and Throat (ENT) clinic with clinical discharge. Hospital based cross sectional study design was implemented for this study.

Sampling technique: Convenient sampling technique was implemented in which patients who had developed ear infection and came to ENT outpatient department with clinically approved ear discharge during the study period were enrolled.

Inclusion and exclusion criteria: Patients who give their consent and had ear discharge with ENT specialist physician diagnosis was included in the study while patients who refuse to participate was excluded from the study.

Study variables: Socio-demographic, age, sex, previous infection, previous treatment history were considered as independent variables while growth of staphylococcus aureus isolates and antimicrobial susceptibility pattern (susceptible, resistance, intermediate) were considered dependent variables in this study.

\section{Broth preparation}

Tryptone soya broth was used as a general purpose media. It was prepared according to the manufacturer's guideline. It was mixed until it completely dissolves on a hot plate and $5 \mathrm{ml}$ was dispensed into each test tube and autoclaved at $1200 \mathrm{C}$ for 15 minutes. 


\section{Sample collection}

Ear swab samples were collected using a sterile swab stick from patients with ear infections at University of Hawassa Comprehensive Specialized Hospital ENT clinic from February 1, 2016 to November 1, 2016. The ear swab sample was sent to the teaching laboratory of Hawassa University Comprehensive Specialized Hospital by Amies transport media (Oxoid Company) for immediate processing. Upon receipt sample was inoculated on Manitol Salt agar and Blood agar (Oxoid Company) plates and incubated at $37{ }^{\circ} \mathrm{C}$ aerobically. The swab was smeared on slide and then gram stained and examined microscopically.

\section{Characterization of isolated bacteria}

After 24 hour incubation, bacteria isolates were characterized based on colonial appearance on Manitol salt agar and blood agar media. Gram stain was done to determine Gram reaction and bacterial morphology. After gram staining those colony shown to be gram positive cocci were subjected to further biochemical tests. Then the grown colony on primary culture media was subcultured on to nutrient agar to get pure colony which is suitable for biochemical test. Biochemical tests carried out were catalase and coagulase tests. Those catalase enzyme negative were considered as coagulase negative Staphylococci and coagulase test positive were considered as $S$. aureus

\section{Antimicrobial sensitivity testing}

Antibacterial susceptibility testing was performed for all isolates according to the criteria of the National Committee for clinical Laboratory Standard (NCCLs) by disc diffusion method[18]. From a pure culture 3-5 selected colonies of bacteria were taken and transferred to a tube containing $5 \mathrm{ml}$ nutrient broth and mixed gently until a homogenous suspension was formed and incubated at $37{ }^{\circ} \mathrm{C}$ until the turbidity of the suspension became adjusted to a McFarnald 0.5. A sterile cotton swab was used and the excess suspension was removed by gentile rotation of the swab against the inside surface of tube. The swab was then used to distribute the bacteria evenly over the entire surface of Mullen-Hinton agar. The inoculated plates were left at room temperature to dry for 3-5 minutes and a set of antibiotic discs such as cloxacillin (CLX) (5), cephalothin (CF) (30), vancomycin (30), amoxicillin-clavulanic acid (AMC) (30), chloramphenicol (C) $(30 \mu \mathrm{g})$, amikacin (AN) (30), Kanamycin (30), Gentamycin (10), and Amoxicillin (2) were dispensed. The inoculated plate then incubated at $37 \mathrm{oC}$ for $24 \mathrm{hrs}$.

\section{Data analysis}

Data was edited, cleaned, entered and analyzed using SPSS version 20. Descriptive analysis such as frequencies and mean was used. Multiple antibiotic resistance (MAR) index was determined by following the procedure described by Krumperman [19]. A MAR index for an isolate is calculated as: number of antibiotics to which isolate is resistant/ total number of antibiotics against which isolate was tested. $P$-value of $<0.05$ was considered statistically significant. The results were presented using tables.

\section{Quality control}

The quality of the data was maintained by strictly implementing quality control measures throughout the whole process of the laboratory work. Staining reagents, culture media and antibiotic discs was checked for their normal shelf life before use. All culture plates was stored at recommended refrigeration temperature $\left(2-8{ }^{\circ} \mathrm{C}\right)$ after prepared and sterilized by autoclaving at $121{ }^{\circ} \mathrm{C}$ for 15 minutes. Antibiotic discs was stored at the same temperature range before use. All laboratory procedures was conducted based on recommended standard laboratory procedures. Preanalytical, analytical and post-analytical stages of quality assurance are strictly followed in accordance to standard operating procedures of the microbiology research laboratory.

\section{Results}

A total of 117 samples were collected from 117 patients with ear discharge. About $56.4 \%$ of the study participant were males. The mean age (SE) of the study participants were 20.4(1.3). The prevalence of S.aureus in our study was $33(28.2 \%)$, in males $21(31.8 \%)$ and females $12(24.5 \%)$. It is found that growth of S.aureus in our sample has not showed association with any of socio-demographic and clinical characteristic variables tested in Table 1.

Overall, S. aureus isolates revealed $3.0-97.0 \%$ level of resistance pattern to the antimicrobials tested. In the determination of the susceptibility of $S$. aureus on nine selected antibiotics by disk diffusion technique, 30(96.8\%) of the isolates were resistant to Cloxacilin and 23(74.2\%) showed resistance to vancomycin (Table 2). A lower level of resistance was observed to cefepime and amikacin.

Multi-drug resistance in this study was taken as resistance to more than one of the antimicrobial drugs tested. Multidrug-resistant (MDR) status of S. aureus isolates was tested against nine classes of antimicrobials. Accordingly, the overall rate of MDR was $100 \%$, all of the isolates was found to be resistant to more than two tested antimicrobials. All of tested S. aureus showed multidrug-resistance shown by MAR index $(0.22$ to 0.66$)$ in Table 3.

\section{Discussions}

In this study, the prevalence of $S$. aureus was found to be $28.2 \%$ which is similar to studies conducted in many different parts of Ethiopia ranging from 24-28 [20-24].In 
Table 1 Socio-demographic characteristics and clinical characteristics of patients with ear discharge at University of Hawassa Comprehensive Specialized Hospital, Hawassa, Ethiopia, February 1, 2016 to November 1, 2016

\begin{tabular}{|c|c|c|c|c|c|}
\hline \multirow[t]{2}{*}{ Variables } & & \multicolumn{2}{|c|}{ Growth of S. aureus } & \multirow[t]{2}{*}{ Total N (\%) } & \multirow[t]{2}{*}{$P$-value } \\
\hline & & Yes (\%) & No (\%) & & \\
\hline \multirow[t]{4}{*}{ Age } & Under 5 & $7(41.2)$ & 10(58.8) & 17 & ref \\
\hline & $6-15$ & 10(31.2) & $22(68.8)$ & 32 & 0.6 \\
\hline & $16-30$ & 13(27.1) & $35(72.9)$ & 48 & 0.6 \\
\hline & Above 31 & $4(20.0)$ & 16(80.0) & 20 & 0.2 \\
\hline \multirow[t]{2}{*}{ Sex } & Male & $21(31.8)$ & $45(68.2)$ & 66 & 0.41 \\
\hline & Female & $12(24.5)$ & $37(75.5)$ & 49 & \\
\hline \multirow[t]{2}{*}{ History of previous infection } & Yes & $25(28.4)$ & $63(71.6 \%)$ & 88 & 0.98 \\
\hline & No & $6(28.6 \%)$ & $15(71.4 \%)$ & 21 & \\
\hline \multirow[t]{2}{*}{ Previous treatment with antimicrobials } & Yes & 21(29.6) & $50(70.4 \%)$ & 71 & 0.88 \\
\hline & No & $11(28.2 \%)$ & $28(71.8 \%)$ & 39 & \\
\hline \multirow[t]{2}{*}{ Is there ear pain } & Yes & $26(27.9)$ & $67(72.1 \%)$ & 93 & 0.98 \\
\hline & No & $9(40.9 \%)$ & $13(59.1 \%)$ & 22 & \\
\hline \multirow[t]{2}{*}{ Is there itching of external ear } & Yes & $21(30.4 \%)$ & $48(69.6 \%)$ & 69 & 0.20 \\
\hline & No & $5(17.9 \%)$ & $23(82.1 \%)$ & 28 & \\
\hline \multirow[t]{2}{*}{ Is there hearing problem } & Yes & $14(26.4 \%)$ & $39(73.6 \%)$ & 53 & 0.71 \\
\hline & No & $14(29.8)$ & $33(70.2)$ & 47 & \\
\hline \multirow[t]{2}{*}{ Is there fever } & Yes & $8(25 \%)$ & $24(75 \%)$ & 32 & 0.58 \\
\hline & No & $20(30.3 \%)$ & $46(69.7)$ & 66 & \\
\hline
\end{tabular}

this study, we estimated prevalence of $S$. aureus resistance to nine different antimicrobial agents commonly used in Ethiopia. Overall, the study provided evidence regarding the prevalence of $S$. aureus resistance to different antimicrobial agents based on ear discharge specimens collected from 117 patients.

It was found that $S$. aureus resistance to commonly available antimicrobial agents in Ethiopia was alarmingly high. The resistance to most preferred agents to S.auerus infection like methicillin and Vancomyicn have dangerously increased compared to previous similar studies [20-22, 25, 26]. About $96.8 \%$ of S.aureus was cloxacillin/methicillin resistant (MRSA) and $74.2 \%$ was vancomycin resistant (VRSA). VRSA rattles clinicians, since there is no drug of choice to treat life-threatening infections with MRSA.

The implication of high prevalence of MRSA and resistance to vancomycin, urges for better second-line drugs for suspected or verified $S$. aureus infections and surgical prophylaxis. Second-line drugs are more expensive and, because of their adverse effects, monitoring during treatment is advisable which increases the costs even further.

S. aureus resistance particularly MRSA in this study is higher than many of similar studies conducted in Ethiopia

Table 2 Antimicrobial resistance patterns of s. aureus isolates $(n=33)$ from ear discharges of study participants, at University of Hawassa comprehensive specialized hospital February 1, 2016 to November 1, 2016

\begin{tabular}{lllll}
\hline & Antimicrobial agents & Sensitive N (\%) & Intermediate N (\%) & Resistant N (\%) \\
\hline 1 & Amoxicillin-clavulanic acid & $13(39.4)$ & $4(12.0)$ & $16(48.5)$ \\
3 & Cefepime & $26(78.8)$ & $5(15.2)$ & $1(3.0)$ \\
4 & Gentamycin & $26(78.8)$ & $1(3.0)$ & $6(18.2)$ \\
5 & Amikacin & $32(97.0)$ & 0 & $1(3.0)$ \\
6 & Vancomycin & $8(24.2)$ & $2(6.1)$ & $23(74.2)$ \\
7 & Cloxacilin/Methicillin & $1(3.0)$ & 0 & $30(97.0)$ \\
8 & Kanamycin & $29(87.9)$ & 0 & $4(12.1)$ \\
9 & Cephalothin & $27(81.8)$ & 0 & $6(18.2)$ \\
\hline
\end{tabular}


Table 3 Multidrug resistance profiles of $S$. aureus isolates to the tested antibiotics $(n=9)$ between February 1, 2016 to November 1, 2016 in University of Hawassa comprehensive specialized hospital

\begin{tabular}{|c|c|c|c|}
\hline & Parameter & Frequency & MAR index \\
\hline 1 & $\mathrm{R} 2=\operatorname{Van}, \mathrm{Amx}$ & 1 & 0.22 \\
\hline 2 & $\mathrm{R} 2=\mathrm{Amx}, \mathrm{Cxc}$ & 3 & 0.22 \\
\hline 3 & $\mathrm{R} 3=\mathrm{Gen}, \mathrm{Amx}, \mathrm{Cxc}$ & 2 & 0.33 \\
\hline 4 & $\mathrm{R} 3=\operatorname{Van}, \mathrm{Amx}, \mathrm{Cxc}$ & 5 & 0.33 \\
\hline 5 & R3 = Aug, Van, Amx & 1 & 0.33 \\
\hline 6 & $\mathrm{R} 3=$ Aug, Amx, Cxc & 1 & 0.33 \\
\hline 7 & $\mathrm{R} 3=\mathrm{Amx}, \mathrm{C} \times \mathrm{C}, \mathrm{Clt}$ & 1 & 0.33 \\
\hline 8 & R4 = Aug, Van, Cxc,Amx & 2 & 0.44 \\
\hline 9 & $\mathrm{R} 4=\mathrm{Van}, \mathrm{Amx}, \mathrm{Kan}, \mathrm{CxC}$ & 2 & 0.44 \\
\hline 10 & $\mathrm{R} 4=\mathrm{Van}, \mathrm{Amx}, \mathrm{Cxc}, \mathrm{CLT}$ & 1 & 0.44 \\
\hline 11 & $\mathrm{R} 4=$ Van, Amx, Gen, Cxc & 1 & 0.44 \\
\hline 12 & R4 = Aug, Van, Amx, Cxc & 6 & 0.44 \\
\hline 13 & R5 = Aug,Van, Amx,Cxc,Clt & 3 & 0.55 \\
\hline 14 & R6 = Aug, Van, Amx, Kan,Cxc, Gen & 2 & 0.66 \\
\hline 15 & R6 $=$ Van, Amx, Kan, Cxc, Clt, Gen & 1 & 0.66 \\
\hline 16 & R6 = Aug,Van,Gen,Cxc,Kan,Amx & 1 & 0.66 \\
\hline
\end{tabular}

*Aug = amoxacilin-clavulanic acid, Van = Vancomycin, Amx = Amoxacilin, $\mathrm{Cxc}=$ cloxacilin, Kan = Kanamycin, Gen = gentamycin, Clt = Cephallothin, $\mathrm{Cfp}=$ cefepime, Amk = Amikacin

*MAR index $=$ multiple antibiotic resistance index $=$ no. of antimicrobials to which the isolate is resistant/no. of antibiotics to which the isolate is subjected

$[17,20,23]$. This indicates a rapid rise in MRSA. The WHO report shows MRSA prevalence between 33\%-95\% in Africa, 43\%-45\% in America, 13\%-18\% in Eastern Mediterranean Region, $27 \%-50 \%$ in Europe, $2 \%-80 \%$ in the South-East Asia region, and $4 \%-84 \%$ in the Western Pacific Region [27]. Our finding is much higher than the WHO report and calls greater attention to the threat.

High resistance suggests that the origin of the isolates were from highly resistant source where antimicrobials are often used. Global pattern of AMR shows variation among different geographic, socioeconomic strata and studies [27-29]. Variation may be to differences in time, place, design, and population involved in the study. Variation may be due to healthcare facilities conditions like implementation and monitoring of infection prevention policies and rationale antimicrobial usage which varies in different facilities. Studies are often conducted within a specified time and locality. It is reasonable to assume population under study might be infected by the same strains of the agent at specified period of time and location.

This study revealed aminoglycosides (amikacin and kanamycin) and fourth generation cephalosporin cefepime showed lower level of resistance. This may be due to rare use/availability of aminoglycosides (amikacin and kanamycin) in the treatment of infections in Ethiopia. Although other aminoglycosides (gentamycin and streptomycin) commonly available in Ethiopia. common use of gentamycin and streptomycin may not contribute to emergence of resistance to amikacin and kanamycin due to low crossresistance among aminoglycosides [30]. Cefepime is totally absent in Ethiopia. It appears that amikacin, kanamycin and cefepime more effective antimicrobials in the treatment of $S$. aureus infections in Ethiopia

Many factors contribute to AMR. First, lack of infection prevention contributes to recurrent infection then to spread of resistant strains. Second, misuse of antimicrobials from prescription-dispensing-to patient use [31]. In Ethiopia, it is a common practice that antibiotics can be purchased without prescription, which leads to misuse of antibiotics by the public [32]. Third factor could be misuse of antibiotics by health professionals and non-standardized practice [31]. The fourth factor could be poor hospital hygienic conditions [33].

Lack of culture and routine antimicrobial susceptibility testing which diverts to empiric therapy may also contribute to the spread [28]. In line to strategies for prevention and containment of $S$. aureus there is a need for innovative way of halting AMR. Combination therapy, design of new drugs and availability of alternative antimicrobial agents will play important role in fighting against AMR to $S$. aureus.

Multi-drug resistant S. aureus isolates to antimicrobials was alarmingly high in this study in which resistance up to six drug was recorded. Empirical prophylaxis and treatment needs careful selection of effective drugs. It needs continuous monitoring of the antimicrobial susceptibility surveillance system of $s$. aureus for the selection of appropriate therapy, developing the antimicrobials policy and limiting the use of reserve antibiotics. Strict consideration for $s$. aureus infection and proper usage of antimicrobials policy are recommended in decreasing the incidence and occurrence of multidrug resistant $s$. aureus infections.

The interpretation of this findings requires considering the limitations thereof. First, this is invitro testing and may not equate to patient outcome. Second, identification of different resistant genes like mecA, mecC and PVL-toxin which provides insight into the distribution of strains and the extent of antimicrobials susceptibility patterns in the area has not been included. Third, the small sample size of the current study may also be a limitation.

\section{Conclusions}

This study reveals that $S$. aureus has gotten frighteningly resistant to many of common antimicrobials used in Ethiopia. It is highly resistant to amoxicillin, vancomycin, amoxicillin -clavulanic acid, Gentamycin, kanamycin, amikacin. Resistance to vancomyin is terrifyingly high. It urges us to include novel drugs in national drug list in Ethiopia 
in order to delay resistance and treat resistant strains. Emergence of VRSA highlights the value of prudent prescribing of antimicrobials (including vancomycin) and avoiding their irrational use. Further researches which focus on identifying dynamics promoting resistance, identifying high risk strains and molecular genetic basis of resistance are needed.

\section{Abbreviations}

AMR: Antimicrobial resistance; ENT: Ear nose throat; MAR: multiple antibiotic resistance; MRSA: Methicillin resistant Staphylococcus aureus; NCCLs: National Committee for clinical Laboratory Standard; S. aureus: Staphylococcus aureus; VRSA: Vancomycin resistant Staphylococcus aureus

\section{Acknowledgments}

The authors need to express their gratitude to Mekedes Taddesse and Sara Taddesse, for their kind support in data collection and experimentation respectively.

\section{Funding}

This research is funded by a grant from University of Hawassa vice president for research, development and technology transfer.

\section{Availability of data and materials}

The datasets during for the current study are available from the corresponding author upon request.

\section{Authors' contributions}

SD, AT conceptualized the research idea. SD, AT and MW prepared the proposal. MW, SD, and AT conducted the experimentation. MB supervised data collection. SD fed the data and performed the analyses. SD prepared the draft manuscript. All authors revised, edited and approved the final manuscript.

\section{Competing interests}

The authors declare that they have no competing interests.

\section{Consent for publication}

Not applicable.

\section{Ethics approval and consent to participate}

The research proposal was reviewed and approved by the Institutional review board of the College of Medicine and Health Sciences of University of Hawassa. Written informed consent of the participants or guardians was obtained after adequate explanation of purpose, importance and potential discomforts of the study. The procedure of specimen collection was explained for all participants.

\section{Publisher's Note}

Springer Nature remains neutral with regard to jurisdictional claims in published maps and institutional affiliations.

\section{Author details}

'Department of Pharmacology, School of Medicine, College of Medicine and Health Sciences, University of Hawassa, P. O. Box 1560, Hawassa, Ethiopia. ${ }^{2}$ Department of medical laboratory technology, College of Medicine and Health Sciences, University of Hawassa, Hawassa, Ethiopia. ${ }^{3}$ Department of ear, nose and throat, School of Medicine, College of Medicine and Health Sciences, University of Hawassa, Hawassa, Ethiopia.

Received: 10 January 2017 Accepted: 4 May 2017

Published online: 15 May 2017

\section{References}

1. John C. Antimicrobial resistance: An update from the Canadian Committee on Antibiotic Resistance. Can J Infect Dis Med Microbiol. 2002;16(5):309-11.

2. Levy SB, O'Brien TF. Global Antimicrobial Resistance Alerts and Implications. Clin Infect Dis. 2005;41:S219-220.

3. WHO: World Health Organization. Department of essential drugs and medicines policy. WHO Workshop on containment of antimicrobial resistance in Europe,
26-27 February 2004 in Wernigerode, Germany. Bundesgesundheitsblatt Gesundheitsforschung Gesundheitsschutz. 2005;48(2):221-31.

4. Gould D, Chamberlaine A. Staphylococcus aureus: a review of the literature. J Clin Nurs. 1995:4(1):5-12.

5. Kirby W. Extraction of a highly potent penicillin inactivator from penicillin resistant staphylococci. Science. 1944;99:452-3.

6. Simpson S. Methicillin resistant Staphylococcus aureus and its implications for nursing practice: a literature review. Nurs Pract. 1992;5(2):2-7.

7. Mulligan ME, Murray-Leisure KA, Ribner BS, Standiford HC, John JF, Korvick JA, Kauffman CA, Yu VL. Methicillin-resistant Staphylococcus aureus: a consensus review of the microbiology, pathogenesis, and epidemiology with implications for prevention and management. Am J Med. 1993;94(3):313-28.

8. Noble WC. Variation in the prevalence of antibiotic resistance of Staphylococcus aureus from human skin and nares. J Gen Microbiol. 1977;98(1):125-32.

9. Smith A, Bagg J. An update on antimicrobial chemotherapy: 2. The mechanisms of antibiotic resistance. Dent Update. 1998;25(5):203-4. 206-208.

10. Dewan ML. Prevalence of Staphylococcus aureus, phage types 71,80 \& 81 among healthy human carriers and their antibiotics resistance. Indian J Public Health. 1974;18(1):8-14.

11. Cuevas O, Cercenado E, Vindel A, Guinea J, Sanchez-Conde M, SanchezSomolinos M, Bouza E. Evolution of the antimicrobial resistance of Staphylococcus spp. in Spain: five nationwide prevalence studies, 1986 to 2002. Antimicrob Agents Chemother. 2004;48(11):4240-5.

12. Hiramatsu K, Cui L, Kuroda M, Ito T. The emergence and evolution of methicillin-resistant Staphylococcus aureus. Trends Microbiol. 2001;9:486-93.

13. Ullauri A, Smith A, Espinel M, Jimenez C, Salazar C, Castrillon R. WHO ear and hearing disorders survey: Ecuador national study 2008-2009. 2014. Science CPi.

14. Alberti PW, Goelzer CH, Hansen H, Sehrndt GA. The pathophysiology of the ear in Occupational Exposure to Noise Evaluation, Prevention and Control. Geneva: WHO; 2001

15. Woodfield G, Dugdale A. Evidence behind the WHO guidelines: hospital care for children: what is the most effective antibiotic regime for chronic suppurative otitis media in children?. J Trop Pediatr. 2008;54(3):151-6.

16. WHO. Chronic suppurative otitis media, burden of illness and management option. Geneva: WHO; 2004. p. 10-47.

17. Abera B, Biadeglegne F. Antimicrobial resistance patterns of Staphylococcus aureus and Proteus spp. isolated from otitis media at Bahir Dar Regional Laboratory, North West Ethiopia. Ethiop Med J. 2009:47(4):271-6.

18. NCLSI. Clinical and Laboratory Standards Institute, Performance standards for antimicrobial susceptibility testing, vol. M100-S124. 2014.

19. Krumperman $\mathrm{PH}$. Multiple antibiotic resistance indexing of Escherichia coli to indentify high-risk sources of fecal contamination of foods. Appl Environ Microbiol. 1983;46:165-70.

20. Hailu D, Mekonnen D, Derbie A, Mulu W, Abera B. Pathogenic bacteria profile and antimicrobial susceptibility patterns of ear infection at Bahir Dar Regional Health Research Laboratory Center, Ethiopia. SpringerPlus. 2016;5:466.

21. Argaw-Denboba A, Abejew AA, Mekonnen AG. Antibiotic-Resistant Bacteria Are Major Threats of Otitis Media in Wollo Area. Northeastern Ethiopia: A Ten-Year Retrospective Analysis. Int J Microbiol. 2016;2016:8724671.

22. Wasihun AG, Zemene Y. Bacterial profile and antimicrobial susceptibility patterns of otitis media in Ayder Teaching and Referral Hospital, Mekelle University, Northern Ethiopia. SpringerPlus. 2015;4:701.

23. Worku M, Bekele M. Bacterial isolate and antibacterial resistance pattern of ear infection among patients attending at Hawassa university referral Hospital, Hawassa, Ethiopia. Indian J Otol. 2014:20:155-9.

24. Abera B, Kibret M. Bacteriology and Antimicrobial Susceptibility of Otitis Media at Dessie Regional Health Research Laboratory, Ethiopia. Ethiop J Health Dev. 2011;25(2):161-7.

25. Shiferaw B, Gelaw B, Assefa A, Assefa Y, Addis Z. Bacterial isolates and their antimicrobial susceptibility pattern among patients with external ocular infections at Borumeda hospital, Northeast Ethiopia. BMC Ophthalmol. 2015;15:103.

26. Negussie A, Mulugeta G, Bedru A, Ali I, Shimeles D, Lema T, Aseffa A. Bacteriological Profile and Antimicrobial Susceptibility Pattern of Blood Culture Isolates among Septicemia Suspected Children in Selected Hospitals Addis Ababa, Ethiopia. Int J Biol Med Res. 2015;6(1):4709-17.

27. WHO. Antimicrobial resistance: global report ton surveillance. France: World Health Organization; 2014.

28. DACA. Antimicrobials use, resistance and containment baseline survey: syntheses of findings. AdisAbaba: DACA; 2009.

29. Falagas ME, Karageorgopoulos DE, Leptidis J, Korbila IP. MRSA in Africa: filling the global map of antimicrobial resistance. PLoS One. 2013;8(7):e68024. 
30. Bang J, Rosdahl VT, Bentzon MW, Rosendal K. Gentamicin-resistant Staphylococcus aureus strains and their susceptibility to other aminoglycosides. Acta Pathol Microbiol Immunol Scand B: Microbiol. 1982; 90(5):361-70.

31. Yadesa TM, Gudina EK, Angamo MT. Antimicrobial Use-Related Problems and Predictors among Hospitalized Medical In-Patients in Southwest Ethiopia: Prospective Observational Study. PLoS One. 2015;10(12):e0138385.

32. Gebretekle GB, Serbessa MK. Exploration of over the counter sales of antibiotics in community pharmacies of Addis Ababa. Ethiopia: pharmacy professionals' perspective. Antimicrob Resist Infect Control. 2016;5:2.

33. Abera B, Kibret M, Mulu W. Knowledge and beliefs on antimicrobial resistance among physicians and nurses in hospitals in Amhara Region, Ethiopia. BMC Pharmacol Toxicol. 2014;15:26.

Submit your next manuscript to BioMed Central and we will help you at every step:

- We accept pre-submission inquiries

- Our selector tool helps you to find the most relevant journal

- We provide round the clock customer support

- Convenient online submission

- Thorough peer review

- Inclusion in PubMed and all major indexing services

- Maximum visibility for your research

Submit your manuscript at www.biomedcentral.com/submit
Biomed Central 